\title{
Resistance to extinction following exposure to a complex situation'
}

Twenty-one rats were trained to run to a sucrose reinforcement. They were then extinguished, retrained, and re-extinguished under conditions wherein each trial was preceded by exposure to a complex situation (Group C), a simple situation (Group P), or no exposure (Group N). Exposure to either a complex or simple situation increased resistance to extinction. During the second extinction phase, Group $C$ performed better than either the $\mathrm{P}$ or $\mathrm{N}$ groups. Response facilitation was attributed to the activating effects of the exposure situations.

It has been shown (Hommel, 1966) that rats exposed to a complex situation prior to a reinforced run to a sucrose solution will increase their speed of running in comparison to rats that were exposed to a simple situation. The explanation offered was that exposure to complexity and/or novelty results in an increase in activation (Malmo, 1959) which is expressed in the vigor of the succeeding response. More generally, it was assumed that reinforcers are activating. This reinforcer-activator hypothesis may serve as an explanation for Anderson's (1941) results in which rats made fewer errors in a multiple $\mathrm{T}$-maze following prefeeding. Both reduced errors and increased speed may be the result of increased activation to some moderate level. Since Anderson's rats did not receive any reinforcement at the end of the $T$-maze, the results suggest that the activating effects of the initial reinforcement can be demonstrated as an increased resistance to extinction. The present study attempted to demonstrate that resistance to extinction of a running response increases when each trial follows exposure to a complex situation.

\section{Method}

The Ss were 21 experimentally naive, Long-Evans pigmented rats, about 30 days old at the onset of training. They were housed three to a cage in the experimental room. This room remained dark between experimental sessions, illuminated during sessions by a single, shaded 25 w bulb.

The apparatus consisted of a straight alley runway and a curiosity box, details of which are described in full elsewhere (Hommel, 1966). Essentially, the runway was $69 \mathrm{in.} \mathrm{long} \mathrm{including} \mathrm{two} \mathrm{16.5-in.} \mathrm{endboxes}$ with guillotine doors to prevent retracing. The drinking tube of a conventional water-bottle containing a $25 \%$ sucrose solution (by weight) was inserted through the back wall of the goal box, directly opposite the runway entrance, 1.5 in. above the floor. Running times were measured by a clock controlled by two light-photocell units, one $10.5 \mathrm{in}$. beyond the starting gate and the other 10 in. inside the goal box. The curiosity box was 10 in. square and $11 \mathrm{in}$. in height, so constructed that different floors were used; one plain and eight complex. The plain floor was painted grey while each of the complex floors had five variously designed wooden forms attached vertically to its grey painted base. The forms were painted in various combinations of white, grey and black. For a representative form, see Hommel (1966).

All Ss were trained to run to the sucrose reinforcement. Each $\mathrm{S}$ was allowed $15 \mathrm{sec}$. in the goal box. The Ss were water deprived $14 \mathrm{hr}$. prior to trial 1-6, but on the 7 th and succeeding daily trials food and water were supplied ad libitum. The running time of each $\mathrm{S}$ was converted into logarithm scores by the formula: $\mathrm{X}=\log _{10}$ (running time in sec +1.00 ) for trials 11-14. The mean logarithm score was computed for these four trials for each $S$ and, on that basis, three groups were formed, seven Ss in each: a normal group (N), a plain floor group (P), and a complex floor group (C). On trials 15-24, the sucrose reinforcement was removed from the goal box, and the extinction procedure was as follows: the N Ss were treated as in the training phase; the $\mathrm{P}$ Ss were given $20 \mathrm{sec}$. of exposure to the plain floor in the curiosity box before being put into the runway startbox; and the $\mathrm{C}$ Ss received $20 \mathrm{sec}$. of exposure to a complex floor before each trial. A different complex floor was used each trial. When all eight complex floors had been used, the first floor to be used was repeated, etc. If any $S$ took longer than $100 \mathrm{sec}$. to start to run, it was removed from the apparatus and given a raw score of $100 \mathrm{sec}$. for that trial. On all extinction trials, the Ss were given $15 \mathrm{sec}$. in the goal box, when a goal box entry had been made. The sucrose reinforcement was replaced in the goal box for trials 25-32 to re-establish the running response. The procedure differed from the original training trials in that the three groups were maintained as in the extinction phase. On trials 33-43, the sucrose reinforcement was again removed and the extinction procedure carried out as before, but with one change: a control for handling in the N Ss. The N Ss were taken from the living cage, held for about $3 \mathrm{sec}$. (it took about 3 sec. to put the Ss of the $C$ and $P$ groups into the curiosity box), then replaced in the living cage. After $20 \mathrm{sec}$. , the $S$ was taken from the cage and put into the startbox as in the original extinction trials.

\section{Results and Discussion}

All of the running time scores were converted to logarithm scores. In each case, the logarithm score was based on the first three significant digits of the 


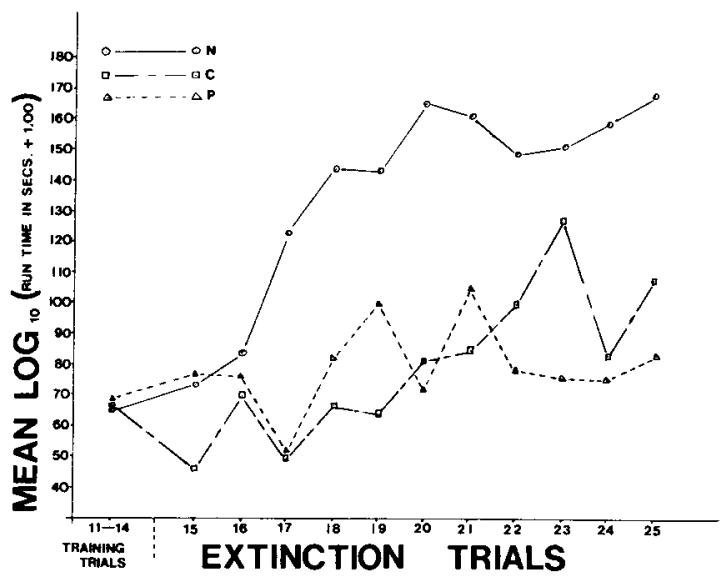

Fig. 1. Extinction following original acquisition. See body of paper for description of $\mathrm{C}, \mathrm{P}$, and $\mathrm{N}$ procedures. (Decimals were omitted from ordinal values.)

running time plus 1.00 . Trials $15-25$ (see Fig. 1) were averaged for each $\mathrm{S}$, and the group means were obtained. Both exposed groups were significantly more resistant to extinction than the normal extinction group ( $P$ vs. $N$ : $t=3.819, \mathrm{df}=12, \mathrm{p}<.01$; C vs. $\mathrm{N}: \mathrm{t}=4.007, \mathrm{df}=12$, $\mathrm{p}<.002$; $\mathrm{P}$ vs. $\mathrm{C}: \mathrm{p}>.10$; all $\mathrm{p}$ values given are twotailed). Surprisingly, tr. re was no significant difference between the two exposed groups. These findings could be the result of the exposure factor, whether simple or complex, or of the extra handling which occurred in the exposure groups but not in the normallyextinguished group.

The re-acquisition data (trials 15-33, notillustrated), showed no significant differences. This disagreement with Hommel's (1966) acquisition results could be due to the masking effect of the $25 \%$ sucrose reinforcement (vs. $8 \%$ in the earlier study).

Analysis of the second extinction phase (trials 34-43) yielded significant differences between all pairs of groups ( $P$ vs. $C: t=2.319, d f=12, p<.05$; $C$ vs. $N$ : $t=2.373, d f=12, p<.05 ; P$ vs. $N: t=4.762, d f=12$, $\mathrm{p}<.002$ ), with the $\mathrm{C}$ group showing the greatest resistance to extinction followed by the P group (see Fig. 2).

Since handling was equated for all three groups during the second extinction phase, the difference produced appears to be a function of exposure to varied degrees of complexity, and not to differences in handling.

The results, in summary, demonstrate: (a) The nonexposed group was more affected by changes in the reinforcement schedule. Exposure, whether to a plain or complex floor, appeared to dampen the differential

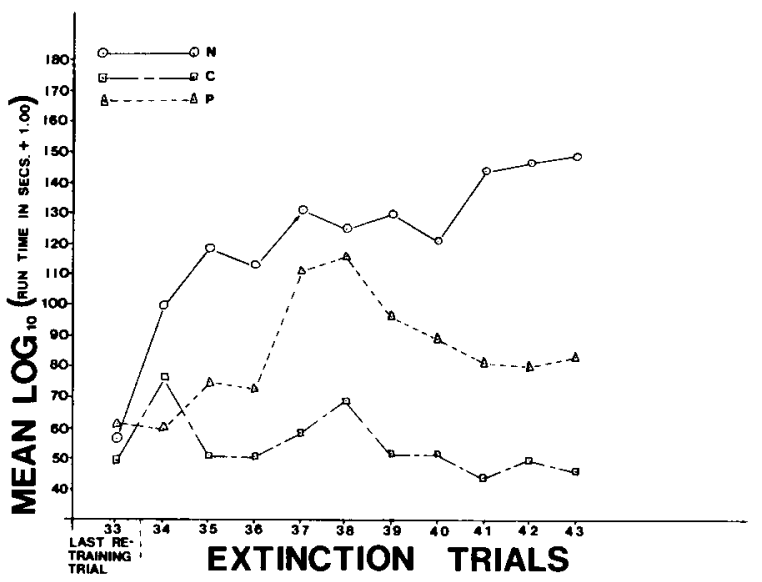

Fig. 2. Extinction following re-acquisition. See body of paper for description of $\mathbf{C}, \mathbf{P}$, and $\mathbf{N}$ procedures during the second extinction phase. (Decimals were omitted from ordinal values.)

effects of acquisition and extinction; (b) While the effect of exposure to the plain floor appeared to deteriorate somewhat during the second extinction phase, exposure to the complex floors increasingly facilitated the response.

The above findings appear to support the hypothesis that exposure to a complex situation is activating. It also appears that exposure to a simple situation produces some activation, especially in the earlier trials. There appears to be a tendency for Ss to habituate to the simple situation upon repetition while the varied complex situations, reasonably, do not show any tendency to lose their effectiveness. These results are apparently related to those of Bacon \& Bindra (1965) in which resistance to extinction was facilitated by varying the color of the startbox.

\section{References}

Anderson, E. E. The externalization of drive IV; The effect of prefeeding on the maze performance of hungry rats. $J$. comp. Psychol., 1941, 31, 349-352.

Bacon, W. E., \& Bindra, D. Stimulus variation and resistance to extinction: satiation or disinhibition? Psychon. Sci., 1965, 2, 119-120.

Hommel, L. S. Increased intensity of a running response following exposure to a visually complex situation. Psychol. Rec., 1966, $16,243-250$.

Malmo, R. B. Activation: A neuropsychological dimension. Psychol. Rev., 1959, 66, 367-386.

\section{Note}

1. This study was supported by a grant from the California State College at Long Beach Foundation to L. S. Hommel. 